\title{
Neue Laborrichtlinie schreibt mehr Qualitätskontrolle vor
}

\begin{abstract}
Die Bundesärztekammer hat bereits 2008 eine neue Richtlinie zur Qualitätssicherung von Laboruntersuchungen erlassen. Diese wird jetzt zum 1. April „scharf geschaltet“ und hat konkrete Auswirkungen für alle Allergologen, die Laboruntersuchungen durchführen.
\end{abstract}

$\mathrm{D}$ ie Qualitätssicherung von medizinischen Laboruntersuchungen wird über das Medizinproduktegesetz und das Eichgesetz geregelt. Diese beauftragen die Bundesärztekammer mit der unmittelbaren Umsetzung der entsprechenden Maßnahmen. In diesem Zusammenhang erlässt die Bundesärztekammer Richtlinien, die für alle Anwender verbindlich sind. Die letzte Richtlinie wurde im Februar 2008 erlassen. Hierin sind eine ganze Reihe von Neuerungen aufgeführt, die zum 1. April 2010 verbindlich für alle Ärztinnen und Ärzte werden, die medizinische Labortests durchführen - egal ob als Laborfachärzte oder als Fachärzte anderer Gebietsgruppen. Dabei ist es unerheblich, ob in der Arztpraxis lediglich ein Streifentest für Urinanalysen durchgeführt wird, oder ob auch speziellere und komplexere Labortests zur Anwendung kommen, wie zum Beispiel die IgE-Diagnostik in der Allergologie.

\section{Qualitätskontrolle: intern obligat, extern optional}

Die Richtlinie hat einen allgemeinen Teil A. In diesem Teil werden die „Rahmenbedingungen" für das Labor (Facharzt für Laboratoriumsmedizin oder Facharztlabor) geregelt. Eine wesentliche Neuerung ist dabei unter anderem die verbindliche Einführung eines Qualitätsmanagementsystems. Dieses findet sich in Kapitel 7 des Teils A näher erläutert.

Im Teil B, dem speziellen Teil der Richtlinie, werden dann zunächst unter B1 quantitative laboratoriumsmedizinische Untersuchungen abgehandelt. Eine quantitative Untersuchung liegt dann vor, wenn ein quantitatives Merkmal bestimmt wird. Ein Merkmal ist dann quantitativ, wenn dessen Werte einer Skala zugeordnet sind, auf der Abstände definiert sind (metrische oder
Kardinalskala). Entscheidend dafür, ob eine Untersuchung quantitativ ist oder nicht, ist die Art und Weise, wie das Ergebnis im Bericht angegeben wird. Ein Beispiel für die Allergologie: Die Bestimmung des Gesamt-IgE ist in jedem Falle eine quantitative Untersuchung.

Für alle quantitativen Untersuchungen ist die Durchführung einer internen Qualitätskontrolle obligat. Hierzu gehört unter anderem die Ermittlung der laboratoriumsinternen Fehlergrenzen. Hierzu werden mit einer Kontrollprobe an mindestens 15 Tagen Kontrollprobenmessungen durchgeführt. Hieraus wird dann nach einer in der Richtlinie angegebenen Formel die Fehlergrenze nach oben und unten berechnet. Es muss dann bei den weiteren laufenden Messserien im Labor mit der entsprechenden Kontrollprobe geprüft werden, ob sich das Ergebnis innerhalb der vorher festgesetzten Toleranzgrenze befindet. Ist dies einmal nicht der Fall, muss auf Fehlersuche gegangen werden. Diese Aspekte sind im Detail geregelt im Teil B1, Abs. 2.

Volltext online
Die aktuelle, „Richt-
linie der Bundes-
ärztekammer zur
Qualitätssiche-
rung labora-
toriumsmedi-
zinischer Leistun-
gen“, deren Be-
stimmungen
jetzt zum 1. April 2010 Gültigkeit er-
langen, wurde bereits am 15. Februar
2008 publiziert: Deutsches Ärzteblatt 105
(7): A341-55. Der Originalbeitrag ist als
PDF-Datei unter www.aerzteblatt.de im
Online-Archiv des Deutschen Ärzteblattes
nach wie vor kostenlos abrufbar.

Darüber hinaus kann bei diesen quantitativen Untersuchungen auch eine externe Qualitätskontrolle erforderlich sein. Diese wird in Form von Ringversuchen durchgeführt. Ringversuche sind obligat für diejenigen Parameter, die in einer speziellen Tabelle B1 aufgelistet sind. Hierzu gehören zurzeit nicht das Gesamt-IgE und auch nicht das allergenspezifische IgE.

Wenn Sie also nach heutigem Stand davon ausgehen, dass die Messung des allergenspezifischen $\mathrm{IgE}$ eine quantitative Messung ist, so sind Sie gehalten als Anwender und Durchführer dieser Untersuchung, eine interne Qualitätskontrolle aufzubauen. Die Teilnahme an Ringversuchen ist nicht obligat.

Selbstverständlich ist die Teilnahme an Ringversuchen zu empfehlen, da diese zu einer Verbesserung der Qualität führen und zwar sowohl in der eigenen Praxis als auch praxisübergreifend. Solche Ringversuche werden von den beiden damit beauftragten Instituten angeboten, dem INSTAND e.V. (www.instandev. de) und dem Referenzinstitut für Bioanalytik der Deutschen Gesellschaft für Klinische Chemie und Laboratoriumsmedizin (DGKL; www.dgkl.de).

\section{IgE-Bestimmung: qualitativ oder quantitativ?}

Demnächst sollen in einem weiteren Teil B2 die qualitativen Testverfahren näher spezifiziert werden. Gegenwärtig gibt es bei der Bundesärztekammer eine Diskussion darüber, ob die Bestimmung des spezifischen IgE ein qualitativer oder ein quantitativer Test ist. Fällt die Entscheidung für qualitativ, dann wird hierfür ebenfalls eine interne Qualitätskontrolle vorgeschrieben werden. Darüber hinaus könnte eine externe Qualitätskontrolle verbindlich werden (Ringversuche).

In jedem Falle ist zu empfehlen, sich mit der neuen Richtlinie vertraut zu machen. Außerdem ist es dringend anzuraten, sich mit den jeweiligen Herstellern der Geräte- und Reagenzien, die im Labor eingesetzt werden, in Verbindung zu setzen. Diese können Hilfestellung geben, sowohl was den Aufbau des internen Qualitätsmanagementsystems anbelangt als auch was die Durchführung der internen Qualitätskontrolle betrifft.

Prof. Dr. Harald Renz, Marburg 